\title{
Does Obesity without Hyperglycemia Delay Wound Healing in an Obese Mouse Model Induced by a High-Fat Diet?
}

\author{
Tamae Urai', Yukari Nakajima², Kanae Mukai³, Kimi Asano², Mayumi Okuwa³, Junko Sugama1, \\ Toshio Nakatani $^{3 *}$ \\ ${ }^{1}$ Advanced Health Care Science Research Unit, Innovative Integrated Bio-Research Core, Institute for Frontier Science Initiative, \\ Kanazawa University, Ishikawa, Japan \\ ${ }^{2}$ Department of Clinical Nursing, Graduate Course of Nursing Science, Division of Health Sciences, Graduate School of Medical \\ Sciences, Kanazawa University, Ishikawa, Japan \\ ${ }^{3}$ Faculty of Health Sciences, Institute of Medical, Pharmaceutical and Health Sciences, Kanazawa University, Ishikawa, Japan \\ Email: *nakatosi@staff.kanazawa-u.ac.jp
}

How to cite this paper: Urai, T., Nakajima, Y., Mukai, K., Asano, K., Okuwa, M., Sugama, J. and Nakatani, T. (2017) Does Obesity without Hyperglycemia Delay Wound Healing in an Obese Mouse Model Induced by a High-Fat Diet? Health, 9, 1660-1679.

https://doi.org/10.4236/health.2017.912122

Received: September 25, 2017

Accepted: November 26, 2017

Published: November 29, 2017

Copyright (c) 2017 by authors and Scientific Research Publishing Inc. This work is licensed under the Creative Commons Attribution International License (CC BY 4.0).

http://creativecommons.org/licenses/by/4.0/

\section{c) (i) Open Access}

\begin{abstract}
It was unclear that wound healing was delayed in obesity without hyperglycemia. The purpose of this study was to compare the wound healing process between obese and non-obese mice without hyperglycemia by attaching a splint. Three-week-old male mice $(\mathrm{C} 57 \mathrm{BL} / 6 \mathrm{~N})$ were fed high-fat diets $(60 \%$ of calories from fat) in the obesity group, and commercial diets in the control group for 15 weeks. Two circular (4 $\mathrm{mm}$ in diameter) full-thickness wounds were made on the dorsal skin. Body weights and serum leptin levels were significantly higher in the obesity group than in the control group until day 15 after wounding. Fasting blood glucose levels before wounding were lower in the obesity group than in a hyperglycemic rodent model. The macrophage infiltration into subcutaneous fat before wounding in the obesity group was negligible. The ratios of the wound area were not significantly different between the two groups. No significant differences were observed in the number of neutrophils or macrophages or new blood vessels and ratio of myofibroblasts or collagen fibers between the two groups. Our results demonstrated that cutaneous wound healing was not delayed in the obesity group without hyperglycemia and macrophage infiltration into the subcutaneous fat and with high serum leptin levels.
\end{abstract}

\section{Keywords}

Obesity, Mouse, Cutaneous Wound Healing, Splint, Inflammation 


\section{Introduction}

Obesity is defined as the excessive accumulation of fat [1] [2]. Obesity is a major intrinsic factor affecting wound healing [3] [4] [5]. Factors related to wound impairments in obesity are wound tension, variations in adipokines, and type 2 diabetes mellitus (DM) [3].

Obese patients have been reported to exhibit tissue hypoperfusion [6]. As intraabdominal pressure is also greater in obese than in non-obese individuals [7], the increased tension at wound edges was due to the high intraabdominal pressure in obese individuals. Tissue oxygen tension in obese patients was previously shown to be low at and near incisions [8], and contributed to wound dehiscence [3] [5]. Circular wounds in the obese mice changed to oval wounds [9]. Tension acted the oval wounds in obese mice. Epithelization and wound contraction between the minor axes of oval wounds may occur for a short time. Therefore, we proposed a new splinting method that prevents tension and maintains the shape of a wound without suturing peri-wound skin [10]. A splint was made from an ostomy skin barrier/wafer that prevented sags and lines on peri-stoma skin. A skin barrier was placed on peri-wound skin. Splints will support the epithelization and wound contraction between the circular wounds. Splints will lighten wound tension. Lighter tension will not delay wound healing. Therefore, we can evaluate that adipokines in obese mice delay wound healing.

Adipose tissue functions not only as a passive energy storage organ, but also as a dynamic endocrine organ [11]. Adipokines are cell signaling proteins secreted by adipocytes [12]. Obese adipose tissue is characterized by adipocyte hypertrophy [12]. In hypertrophied adipocytes, the expression of pro-inflammatory cytokines, tumor necrosis factor alpha (TNF- $\alpha$ ) is up-regulated, whereas that of anti-inflammatory cytokines, adiponectin is down-regulated. Thus, obesity is associated with a state of chronic, low-grade inflammation [3] [11]-[18]. In the advanced stages of obesity, neutrophils and macrophages infiltrate obese adipose tissue. Macrophage-derived TNF- $\alpha$ induces the release of saturated fatty acids from adipocytes via lipolysis, which, in turn, induces inflammatory changes in macrophages. This paracrine loop between adipocytes and macrophages constitutes a vicious cycle, thereby further accelerating adipose tissue inflammation [11] [12]. On the other hand, the decreased expression of adiponectin and increased expression of MCP-1 in adipose tissue contribute to insulin resistance [19] [20] [21], leading to type 2 DM [14] [22].

In clinical settings, not all obese individuals have hyperglycemia [23] [24]. Therefore, two states: obesity with and without hyperglycemia, need to be considered separately. Wound healing delays in individuals with a hyperglycemic state [25] [26] and DM [3]. Transgenic obese and hyperglycemic models, ob/ob mice [27] and db/db mice [28] also delayed cutaneous wound healing [25] [29] [30] [31]. In order to exclude the influence of hyperglycemia on wound healing, transgenic obese and hyperglycemic models [32] [33] were excluded as the subjects of this study. We created a diet-induced obese mouse model (refer to Ani- 
mals and diets); wild-type mice were fed a high-fat diet.

We hypothesized that wound healing is delayed in obese mice because the degree of wound inflammation with obesity is greater and prolongs inflammatory phase. And their blood glucose (BG) levels were lower than those in transgenic obese and hyperglycemic models [25]-[33]. We then investigated the influence of obesity and hypertrophied adipocytes on cutaneous wound healing.

\section{Materials and Methods}

\subsection{Animals and Diets}

Thirty-one 3-week-old male C57BL/6NCrSlc mice weighing 8.2 - 11.6 g were used (Sankyo Labo Service Corporation, Inc., Tokyo, Japan). They were caged in an air-conditioned room with a temperature of $25.0 \pm 2.0$ degrees and lights on from 08.45 to 20.45 hours. Water was given ad libitum. All animal experiments conducted in this study were reviewed and approved by the Kanazawa University Animal Experiment Committee, and performed in accordance with the Guidelines for the Care and Use of Laboratory Animals of Kanazawa University, Japan (AP-153646). Mice were randomly divided into obesity and control groups. The control group $(n=17)$ was fed a commercial diet (CRF-1; Charles River Laboratories International, Inc., Kanagawa, Japan) and the obesity group $(\mathrm{n}=14)$ a high-fat diet (58Y1 Diet Induced Obesity Rodent Purified Diet with 60\% Energy from Fat; PMI Nutrition International, St. Louis, USA) for 15 weeks (the feeding period) (9). The high-fat diet had 1.4-fold the energy and 6.5-fold the fat of the commercial diet fed to the control group (Table 1). In the evaluation of mouse models, fasting blood glucose (FBG) in mice fasted for 14 hours was assessed using GLUCOCARD (GT-1641; ARKRAY, Inc., Kyoto, Japan) at 15 weeks.

\subsection{Wounding Procedure}

Fifteen weeks after feeding with the high-fat or commercial diet, mice were anesthetized with $1.75 \%$ isoflurane (Wako Pure Chemical Industries, Ltd., Osaka, Japan) via an inhalation system. After mice had been anesthetized, the dorsum was shaved completely and hair removal cream was applied. Once mice remained in an anesthetized state without a pain reaction, two circular $(4 \mathrm{~mm}$ in diameter) full-thickness skin wounds were made on the dorsal skin with a Kai

Table 1. Diet composition.

\begin{tabular}{ccc}
\hline component in $100 \mathrm{~g}$ & Control & High-fat \\
\hline Protein & 21.9 & 23.1 \\
Carbohydrate & 55.3 & 25.9 \\
Fat & 5.4 & 34.9 \\
Energy (kcal) & 357 & 510 \\
\hline
\end{tabular}

The control group was fed commercial pellets (CRF-1). The obesity group was fed a high-fat diet (58Y1). 
sterile disposable biopsy punch (Kai Industries, Gifu, Japan). Punch biopsy skin was used to observe macrophages in skin, namely, subcutaneous tissue (refer to the histological procedure and immunohistological staining). The sag in dorsal skin was smoothed out, and a splint (Varicare ${ }^{\varpi}$ Wafer; ConvaTec Japan Inc., Tokyo, Japan) with a hole in the center was fixed on peri-wound skin. The inside lines of splints were marked on the mouse dorsum to maintain the splints in the same position. Wounds were covered with a circular (approximately $1.4 \mathrm{~cm}$ in diameter) hydrocolloid dressing (Tegaderm ${ }^{\oplus}$; $3 \mathrm{M}$ Health Care, Tokyo, Japan) to maintain a moist environment (Figure 1), and the mouse was then wrapped with adhesive bandages (SKINERGATE ${ }^{\mathrm{mw}}$; Nichiban, Tokyo, Japan). These circular dressings and bandages were changed every day. Splints were kept unless they were damaged by wound exudates. The day when wounds were made was designated as day 0 after wounding.

\subsection{Macroscopic Observations}

The wound healing process was observed from day 0 to day 15 after wounding. Wound edges were traced on polypropylene sheets and wound photographs were taken every day. The traces on the sheets were captured with a scanner onto a personal computer using Adobe Photoshop Elements 7.0 (Adobe System Inc., Tokyo, Japan), and wound areas were calculated using the image analysis software ImageJ ver. 1.42q (Wayne Rasband, National Institutes of Health, Bethesda, Maryland, USA). The ratio of the wound area to the initial wound area was calculated every day. Macroscopic wound re-epithelization was estimated by the difference between the total lesion area and wound area still not covered with an epidermis [34] [35]. The ratio of macroscopic wound re-epithelization was calculated on days $7,9,11,13$, and 15 after wounding.

\subsection{Histological Procedure and Immunohistological Staining}

Mice were euthanized by an IP injection of an overdose of pentobarbital sodium on days $3,7,11$, and 15 after wounding. Wounds and the surrounding intact

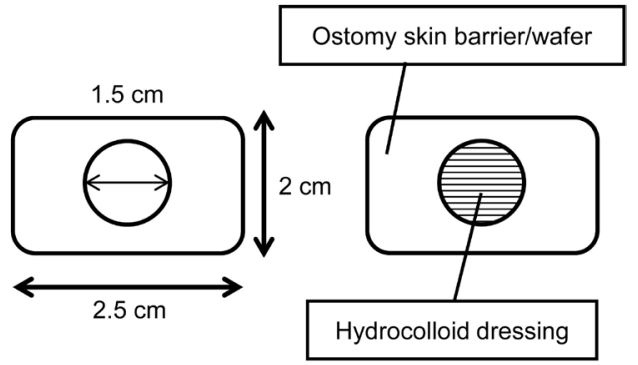

Figure 1. Splint size and wound dressing. The sag in dorsal skin was smoothed out, and a splint (Varicare ${ }^{\circledast}$ Wafer) with a hole in the center was fixed on peri-wound skin. Wounds were covered with a circular (approximately $1.4 \mathrm{~cm}$ in diameter) hydrocolloid dressing (Tegaderm ${ }^{\circledR}$ ) to maintain a moist environment. 
skin were harvested, stapled onto transparent plastic sheets to prevent the over-contraction of specimens, and fixed in $4 \%$ paraformaldehyde in $0.1 \mathrm{~mol} / \mathrm{L}$ phosphate buffer ( $\mathrm{pH}$ 7.4) overnight. Specimens were dehydrated in an alcohol series, cleaned in xylene, and embedded in paraffin to prepare 5 - $\mu$ m-thick serial sections. Each serial section was stained with hematoxylin-eosin (H-E) and Azan, and was also subjected to immunohistochemical staining. The procedure for unmasking antigens was antigen-dependent, as described below.

Immunohistochemistry was performed for neutrophils using a rat monoclonal antibody to neutrophils (Abcam, ab2557, Tokyo, Japan) at a dilution of 1:100, for macrophages using Purified Rat Anti-Mouse Mac-3 (BD Pharmingen, 550292, Tokyo, Japan) at a dilution of 1:100, for $\alpha$-smooth muscle actin ( $\alpha$-SMA) using RbpAb to a-SMA (Abcam, ab5694, Tokyo, Japan) at a dilution of 1:300.

In secondary antibody staining, polyclonal rabbit anti-rat immunoglobulins/ HRP (Dako Denmark A/S, Glostrup, Denmark) at a concentration of 1:300 with 0.003\% mouse serum (Dako Denmark A/S, Glostrup, Denmark) in phosphate-buffered saline (PBS) with $0.1 \%$ Tween-20 was used as anti-neutrophil antibody and anti-mouse Mac-3 antibody, the Dako Envision+ system HRP-labeled polymer anti-rabbit (ready to use) (Dako North America, California, USA) was used as an anti- $\alpha$-SMA antibody.

The process applied was the same for all immunohistochemical stains. In accordance with a previous study [36], after deparaffinization and rehydration, antigen unmasking was accomplished by heating slides in a water bath followed by an incubation in sodium citrate buffer $(10 \mathrm{mM}$ sodium citrate, $0.05 \%$ Tween 20 , $\mathrm{pH}$ 6.0) at approximately $100^{\circ} \mathrm{C}$ for 20 minutes. Slides were washed with PBS with $0.1 \%$ Tween-20 and then incubated with each antibody at $4{ }^{\circ} \mathrm{C}$ overnight. In order to detect primary antibodies, slides were incubated with the secondary antibody with HRP at room temperature for 30 minutes, and then incubated in the Dako Liquid DAB+ Substrate Chromogen System (Dako North America, California, USA) (brown chromogen) at room temperature for 5 minutes or until staining was detected. Light hematoxylin counterstaining was applied for 1 minute for the visualization of cell nuclei. Negative controls were obtained by omitting each primary antibody.

\subsection{Microscopic Observations}

Images were obtained using a digital microscopic camera (DP2-BSW, Olympus Corporation, Tokyo, Japan). The distance between the edges of the wound and the length of the new epithelium were measured, and the latter was divided by the former (length of the new epithelium/total wound length; the epithelization ratio). To analyze neutrophils, macrophages, and a-SMA-positive cells in the granulation tissue, their respective positive cells were counted in three wound areas at a magnification of $\times 40$ using ImageJ to determine their numbers per $\mathrm{mm}^{2}$ on days $3,7,11$, and 15 after wounding. The average number of macrophages in non-wounded skin was counted at 40× magnification in 1 - 3 areas 
(right, center, and left areas in subcutaneous adipocytes), and each number was calculated per $\mathrm{mm}^{2}$. The ratio of collagen (which was stained blue) was expressed as blue color pixels in the wound/total wound pixels using Adobe Photoshop Elements 7.0. The number of blue pixels was divided by the total number of pixels within the wound area. These histological evaluations were performed as described in a previous study [37].

\subsection{Serum Leptin Levels}

Mice were euthanized on days 3, 7, 11, and 15 after wounding. Serum was prepared from blood collected from each mouse through cardiac puncture. Blood was left to stand at room temperature for 60 minutes, then stored at $4^{\circ} \mathrm{C}$ for 4 hours. It was then centrifuged at $3000 \mathrm{rpm}$ for 15 minutes $\left(4^{\circ} \mathrm{C}\right)$ and stored at $-20^{\circ} \mathrm{C}$ until assayed. Serum leptin levels were measured by ELISA (Biovendor Laboratory Medicine, the Czech Republic, COSMO BIO CO., LTD.) according to the manufacturer's guidelines.

Briefly, 96-well microplates pre-coated with the anti-mouse leptin antibody were used. Samples from the obesity group (days 3 and 7 after wounding) and control group (day 3 after wounding) were diluted 20x with Dilution Buffer just prior to the assay. On the other hand, samples from the obesity group (days 11 and 15 after wounding) and control group (days 7, 11, and 15 after wounding) were diluted $2 \times$ with Dilution Buffer. Standard wells included $100 \mu \mathrm{L}$ of serially diluted concentrations of mouse leptin: 4000, 2000, 1000,400, 200, 100, and 0 pg/mL. Sample wells included $100 \mu \mathrm{L}$ of diluted serum samples. The plate was then covered with adhesive film and incubated at room temperature for 1 hour with shaking at ca. $300 \mathrm{rpm}$ on an orbital microplate shaker. All wells were automatically washed four times with washing buffer, followed by the addition of $100 \mu \mathrm{L}$ of the biotin-labeled polyclonal anti-mouse leptin antibody to the wells, and the plate was then covered with adhesive film and incubated with the captured leptin at room temperature for 1 hour. All wells were automatically washed four times with washing buffer, followed by the addition of $100 \mu \mathrm{L}$ of the streptavidin-HRP conjugate. After a 30-minute incubation and the last washing step, the remaining conjugate was allowed to react with $100 \mu \mathrm{L}$ of the substrate solution (tetramethylbenzidine) and the plate was incubated at room temperature for 10 minutes. The reaction was stopped by the addition of $100 \mu \mathrm{L}$ of an acidic solution and the absorbance of the resulting yellow product was measured using a microplate reader (MTP-310Lab, CORONA ELECTRIC Co., Ltd., Ibara$\mathrm{ki}$, Japan) at $450 \mathrm{~nm}$ and $570 \mathrm{~nm}$. Absorbance was proportional to the concentration of leptin.

\subsection{Statistical Analysis}

Data are presented as the mean \pm standard deviation. Data were assessed using unpaired $t$-test comparisons between the obesity and control groups with a commercially available statistics package (JMP ${ }^{\oplus}$ 8.0.1; SAS Institute Inc., Cary, 
NC, USA). Differences were considered significant at $p<0.05$.

\section{Results}

\subsection{Evaluation of the Obesity Status}

Fifteen weeks after feeding, the final body weight in the obesity group was approximately $42 \%$ heavier than that in the control group $(p<0.01)$ (Table 2$)$. FBG levels at week 15 after feeding in the obesity group were significantly higher than those in the control group $(p<0.01)$ (Table 2$)$. The FBG level in the obesity group, a mean of $186.8 \pm 19.0 \mathrm{mg} / \mathrm{dl}$, was lower than that in a diabetic rodent model such as ob/ob mice and GK rats $(295 \pm 66.8$ and $229 \mathrm{mg} / \mathrm{dl}$, respectively; industry data, Japan SLC, Inc., Hamamatsu, Japan). Although body weights in the obesity group gradually decreased after wounding, they were still significantly higher than those in the control group for 15 days after wounding (Figure $2)$. On day 15 after wounding, mean body weight in the obesity group was $33.6 \pm$ $2.9 \mathrm{~g}$, while that in the control group was $26.6 \pm 1.3 \mathrm{~g}(p<0.01)$ (Figure 2).

\subsection{The Ratio of the Wound Area}

Wounds in the obesity group maintained the circular shape (Figure 3(a)). Wound areas in the obesity group peaked on day 4, while those in the control

Table 2. Initial and final body weights (BW) and fasting blood glucose (FBG) levels.

\begin{tabular}{ccc}
\hline Parameter & Control & Obesity \\
\hline Initial BW $(\mathrm{g})$ & $9.4 \pm 1.1$ & $10.4 \pm 0.9^{*}$ \\
Final BW $(\mathrm{g})$ & $32.9 \pm 2.5$ & $46.8 \pm 2.8^{\star *}$ \\
FBG $(\mathrm{mg} / \mathrm{dL})$ & $154.8 \pm 24.5$ & $186.8 \pm 19.0^{* *}$ \\
\hline
\end{tabular}

Body weights were calculated as initial (upon the initiation of the control or high-fat diet) and final weights (the day of making wounds) in each group. Fasting blood glucose levels were assessed at week 15 after feeding. $\mathrm{n}=17$ in the control group and $\mathrm{n}=14$ in the obesity group (Initial and Final BW), and $\mathrm{n}=9$ in the control and obesity groups (FBG). Unpaired $t$-test. ${ }^{*} p<0.05,{ }^{* *} p<0.01$.

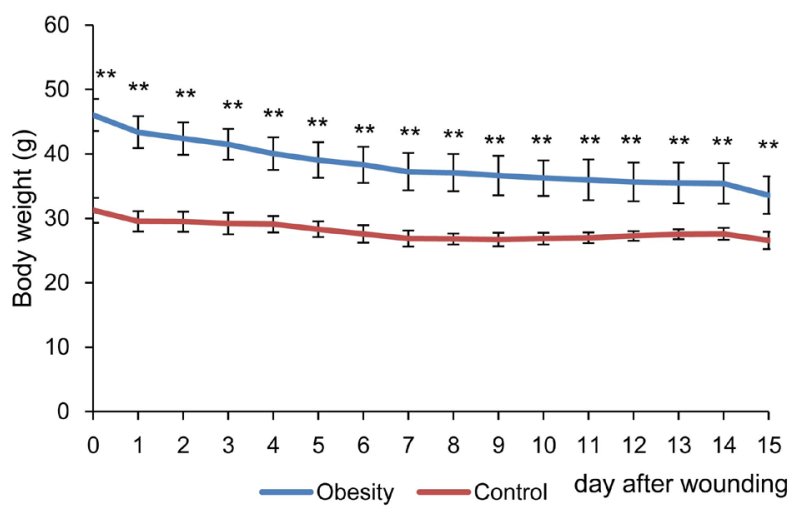

Figure 2. Body weights after wounding. Body weights after wounding are shown on line graphs. Control group $n=6$, Obesity group $n=5$. Values are means, with their standard deviation represented by vertical bars. The mean value was significantly different from that in the control group (unpaired $t$-test comparisons: ${ }^{* *} p<0.01$ ). 


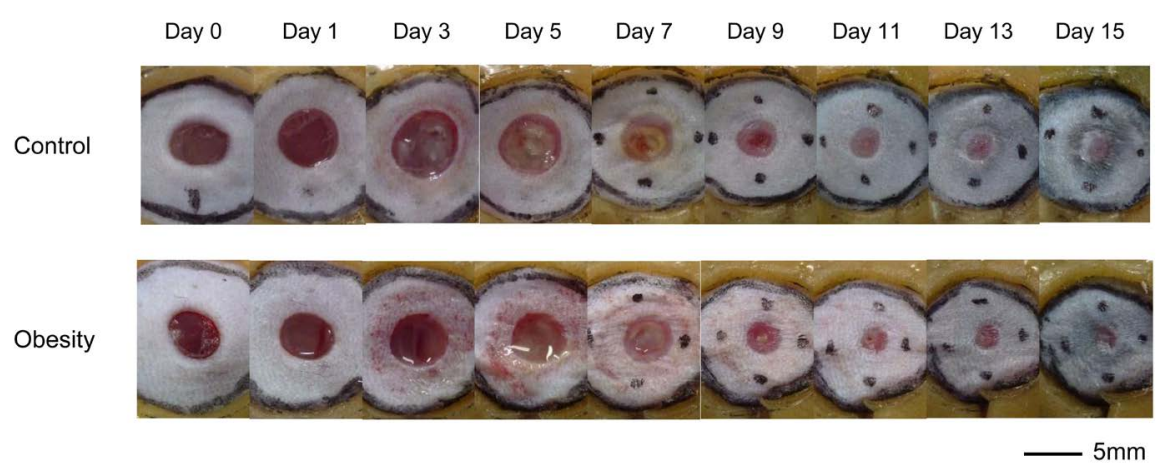

(a)

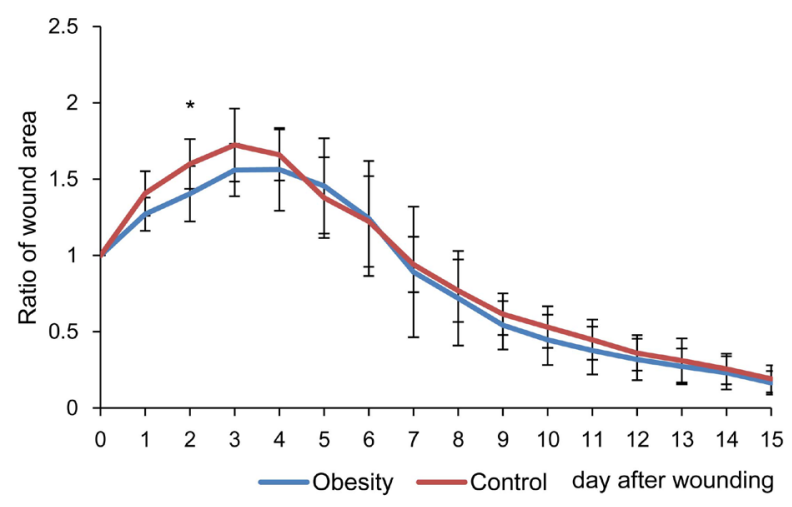

(b)

Figure 3. Macroscopic wound healing. (a) Wounds that were $4 \mathrm{~mm}$ in diameter were made, and the healing process was recorded by photography. Bar, $5 \mathrm{~mm}$. (b) The ratio of the wound area. The ratio was calculated as the wound area on each day divided by that on day 0 . The value on day 0 was the baseline. Control group $n=11$, Obesity group $n=$ 10. Values are means, with their standard deviation represented by vertical bars. The mean value was significantly different from that in the control group (unpaired $t$-test comparisons: $\left.{ }^{\star} p<0.05\right)$.

group peaked on day 3; therefore, the day at which the wound area reached its maximum was similar in the two groups. On days $1-4$, the ratio of the wound area was lower in the obesity group than in the control group (day 2: $p<0.05$ ). On day 15 after wounding, all wounds formed scars. The ratio of the wound area in the obesity group on day 15 was $0.16 \pm 0.08$, while that in the control group was $0.19 \pm 0.09(p=0.83$ ) (Figure $3(b))$. The wound healing process for 15 days appeared to be similar in both groups.

\subsection{Re-Epithelization}

The new epithelium gradually covered the wound surface as wound healing progressed in both groups. On day 11 after wounding, the ratio of re-epithelization in the obesity group was $100 \%$, namely, re-epithelization was completed. The ratio of re-epithelization in the control group was also $100 \%$ (Figure 4(a)). The ratio of macroscopic re-epithelization in the two groups was $100 \%$ on day 13 after wounding (Figure $4(\mathrm{~b})$ ). The re-epithelization was not significantly different between the two groups. 


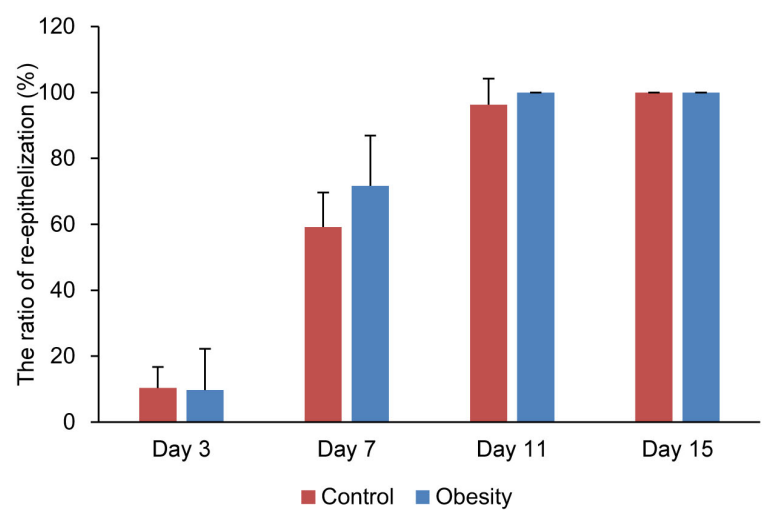

(a)

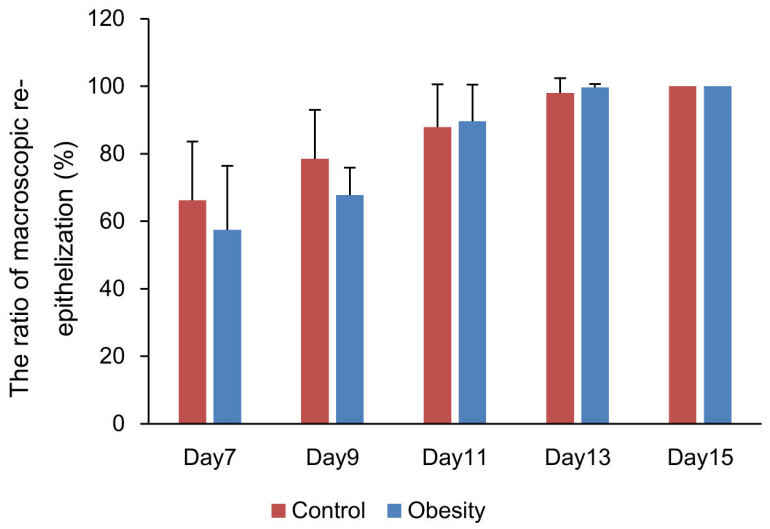

(b)

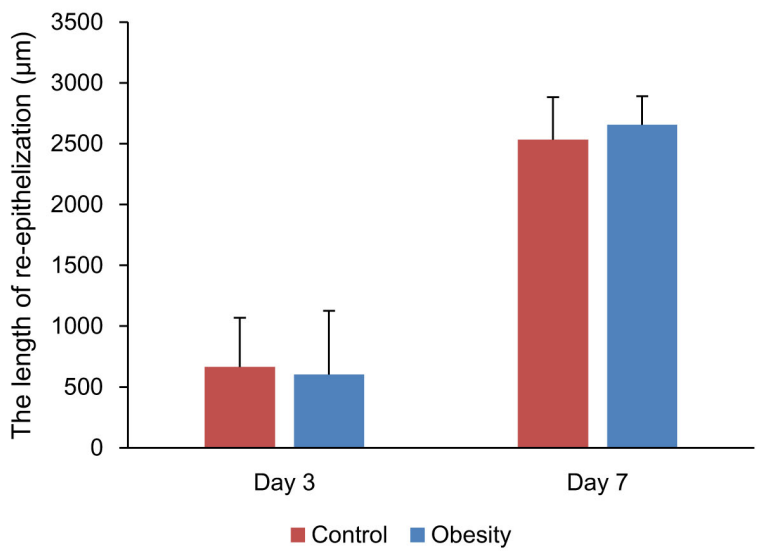

(c)

Figure 4. Macroscopic wound healing and evaluation of re-epithelization. (a) The ratio of re-epithelization. The ratio of re-epithelization was calculated as new epidermis length divided by wound length. (b) The ratio of macroscopic wound re-epithelization. Macroscopic wound re-epithelization was macroscopically estimated by the difference between the total lesion area and wound area still not covered with an epidermis. (c) The length of re-epithelization. The length of re-epithelization was calculated as new epidermis length. (a) Control group $\mathrm{n}=5,5,6$, and 11, Obesity group $\mathrm{n}=6,5,6$, and 10 (on days $3,7,11$, and 15 after wounding, respectively). (b) Control group $n=10$, Obesity group $n=9$ (on days $7,9,11,13$, and 15 after wounding). (c) Control group $n=5,5$, Obesity group $n=6$, 5 (on days 3 and 7 after wounding, respectively). Values are means, with their standard deviation represented by vertical bars. 


\subsection{Neutrophils and Macrophages}

In non-wounded skin, the number of macrophages was $54.5 \pm 27.0 \mathrm{cells} / \mathrm{mm}^{2}$ in the control group $(\mathrm{n}=4)$ and $85.6 \pm 62.7$ cells $/ \mathrm{mm}^{2}$ in the obesity group $(\mathrm{n}=5)$ (Figure 5(a)), with no significant difference being observed between the two groups $(p=0.39)$. On day 3 after wounding, a large number of macrophages were found in wounds in the two groups, and did not markedly change from the number observed on day 7 after wounding. No significant differences were noted in the numbers of macrophages between the two groups in non-wounded skin or in wounded skin on days 3 and 7 after wounding (Figure 5(b) and Figure $5(\mathrm{c})$ ).

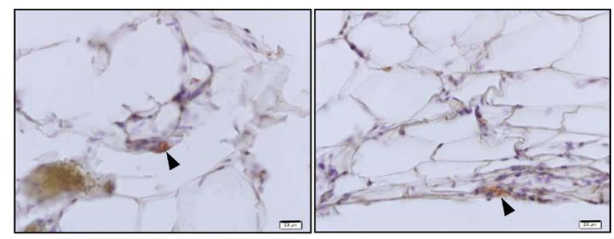

(a)

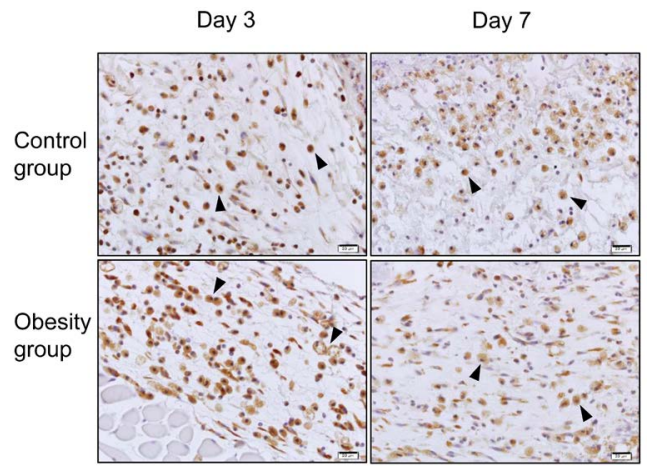

(b)

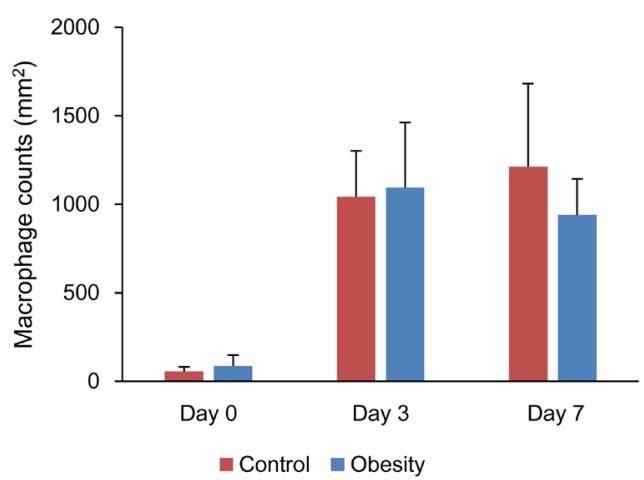

(c)

Figure 5. Macrophages. (a) Macrophages (arrow heads) stained with an anti-Mac-3 antibody were observed in non-wounded skin and (b) wound tissue on days 3 and 7. Bar, 20 $\mu \mathrm{m}$. (c) Number of macrophages per $\mathrm{mm}^{2}$. Control group $\mathrm{n}=4,6,5$, Obesity group $\mathrm{n}=5$, 6,6 (on days 0,3 , and 7 after wounding, respectively). Values are means, with their standard deviation represented by vertical bars. 
On day 3 after wounding, a large number of neutrophils were also detected in wounds in the two groups, and this number decreased on day 7 after wounding. No significant differences were observed in the numbers of neutrophils between the two groups on days 3 and 7 after wounding (Figure 6(a) and Figure 6(b)).

\subsection{Angiogenesis, Collagen Deposition, and Wound Contraction}

The number of new blood vessels peaked on day 11 after wounding in both groups. On day 15 after wounding, the number of new blood vessels in each group slightly decreased (Figure 7 (a) and Figure $7(\mathrm{~b})$ ). The ratio of myofibroblasts peaked on day 11 after wounding in the obesity group and on day 7 after wounding in the control group. The ratio of myofibroblasts in each group peaked on different days (Figure 7 (a) and Figure $7(\mathrm{c})$ ). The ratio of collagen fibers increased gradually from days 7 to 15 after wounding (Figure 8(a) and Figure 8(b)).

No significant differences were observed in the number of new blood vessels or ratios of myofibroblasts and collagen fibers between the two groups on days 7 , 11 , and 15 after wounding.

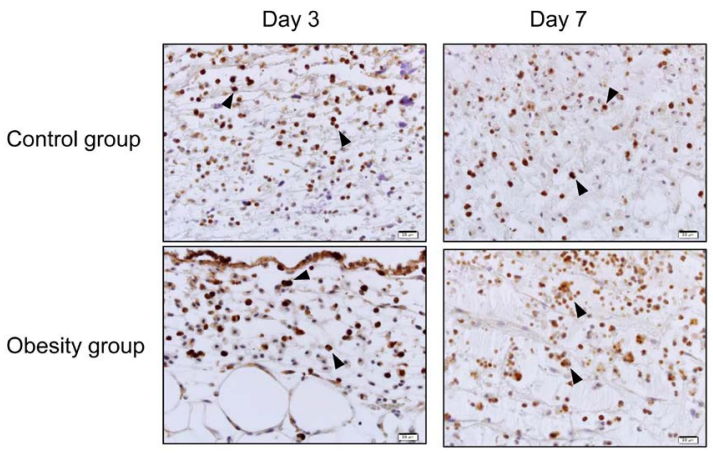

(a)

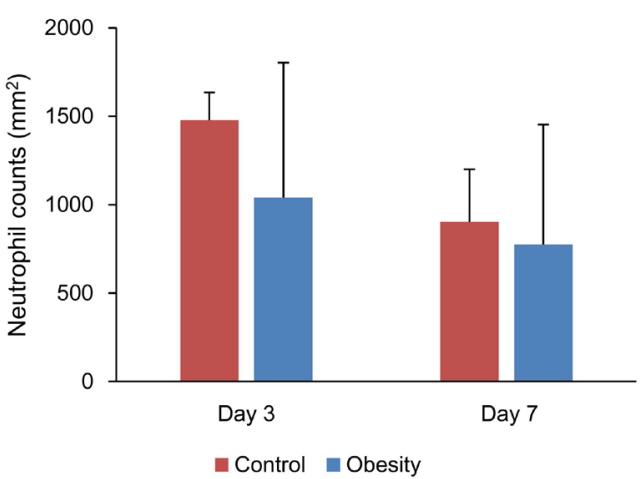

(b)

Figure 6. Neutrophils. (a) Neutrophils (arrow heads) stained with an anti-neutrophil antibody were observed in wound tissue on days 3 and 7. Bar, $20 \mu \mathrm{m}$. (b) Number of neutrophils per $\mathrm{mm}^{2}$. Control group $\mathrm{n}=6$ and 5 , Obesity group $\mathrm{n}=6$ and 6 (on days 3 and 7 after wounding, respectively). Values are means, with their standard deviation represented by vertical bars. 


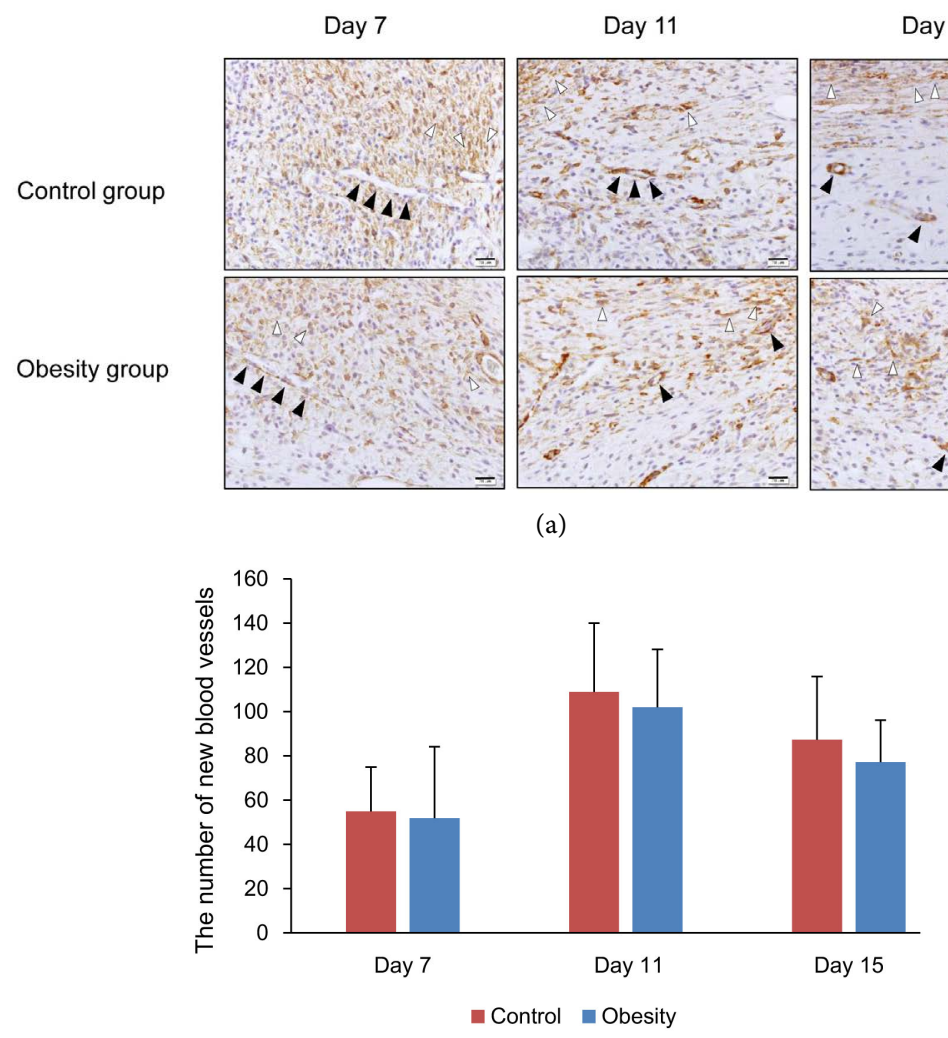

(b)

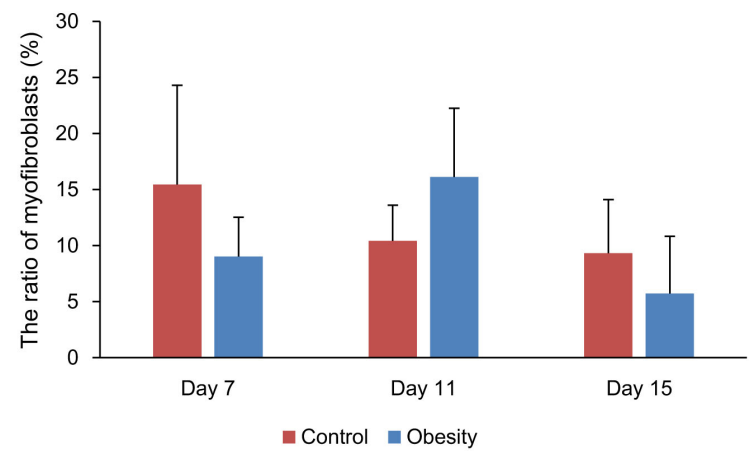

(c)

Figure 7. New blood vessels and wound contraction. (a) New blood vessels (black arrow heads) stained with an anti- $\alpha$-SMA antibody on days 7,11 , and 15. Myofibroblasts (white arrow heads) stained with the anti- $\alpha$-SMA antibody on days 7,11 , and 15 . Myofibroblasts appeared at wound edges on granulation tissue. Insets highlight regions of the wound edge on granulation tissue at a higher magnification. Bar, $20 \mu \mathrm{m}$. (b) The number of new blood vessels per $\mathrm{mm}^{2}$ and (c) the ratio of myofibroblasts (\%) are shown on box graphs. (b), (c) Control group $n=5,5$, and 6, Obesity group $n=6,6$, and 9 (on days 7, 11, and 15 after wounding, respectively). Values are means, with their standard deviation represented by vertical bars.

\subsection{Serum Leptin Levels}

On day 3 after wounding, serum leptin levels were significantly higher in the obesity group $(30.5 \pm 3.5 \mathrm{ng} / \mathrm{ml})$ than in the control group $(6.7 \pm 3.8 \mathrm{ng} / \mathrm{ml})$. On day 7 after wounding, serum leptin levels in the obesity group markedly de- 


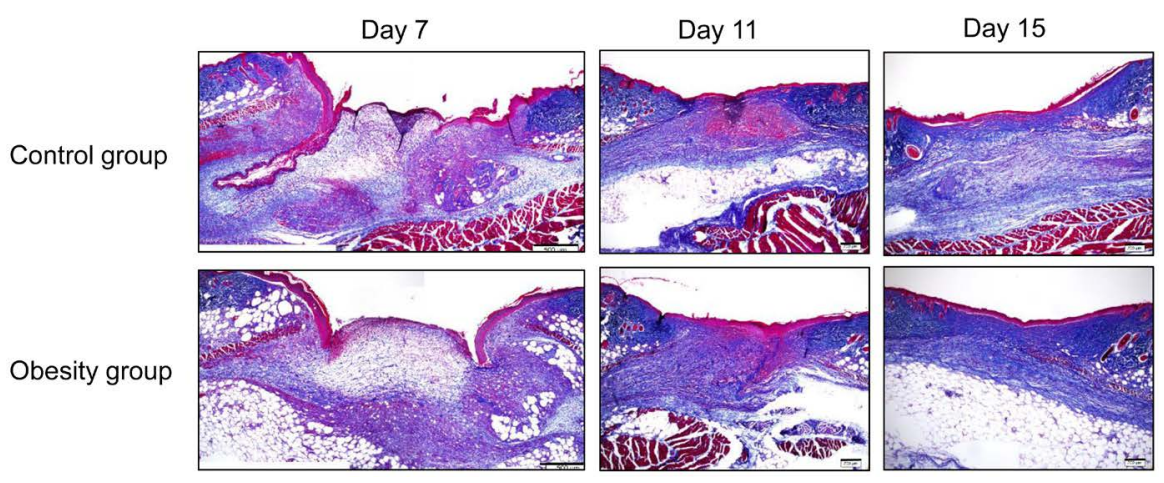

(a)

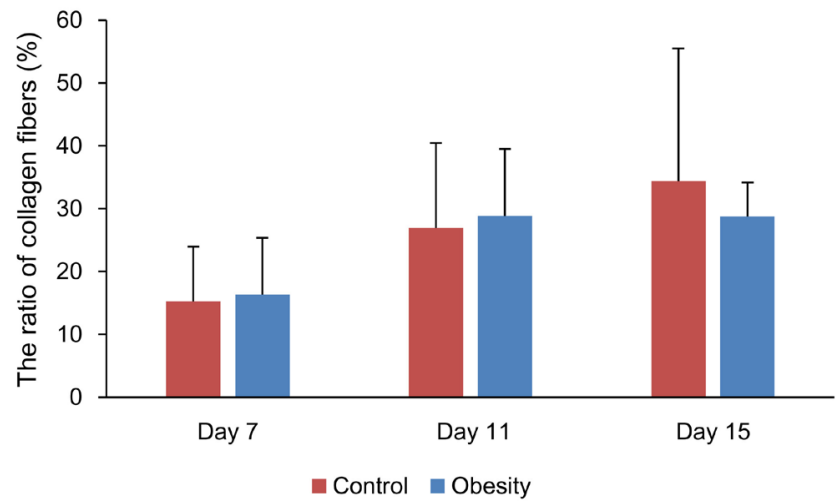

(b)

Figure 8. Collagen fibers. (a) Azan staining on days 7, 11, and 15. Collagen fibers, which stained blue, were observed in granulation tissue. Bars, $500 \mu \mathrm{m}$ (day 7) and $200 \mu \mathrm{m}$ (days 11 and 15). (b) The ratio of collagen fibers (\%) is shown on box graphs. Control group $\mathrm{n}$ $=5,6$, and 6 , Obesity group $n=6,6$, and 7 (on days 7,11 , and 15 after wounding, respectively). Values are means, with their standard deviation represented by vertical bars.

creased to $12.5 \pm 3.7 \mathrm{ng} / \mathrm{ml}$; however, this level was significantly higher than that in the control group $(2.8 \pm 1.1 \mathrm{ng} / \mathrm{ml})$. Although serum leptin levels in the obesity group decreased gradually by days 11 and 15 after wounding (day 11: $7.8 \pm$ $0.9 \mathrm{ng} / \mathrm{ml}$, day 15: $4.5 \pm 2.2 \mathrm{ng} / \mathrm{ml}$ ), they were significantly higher than those in the control group (day 11: $3.3 \pm 1.3 \mathrm{ng} / \mathrm{ml}$, day 15: $1.4 \pm 0.8 \mathrm{ng} / \mathrm{ml}$ ). Serum leptin levels were significantly higher in the obesity group than in the control group until day 15 after wounding (Figure 9).

\section{Discussion}

We previously investigated circular (diameter of $6 \mathrm{~mm}$ ) full-thickness skin wound healing in diet-induced obese mice, and found no significant differences in the progression of wound healing between obese and non-obese mice [9]. We identified two factors that contributed to wound healing in obese mice; wound tension and stress of wounding.

In our previous study [9], circular wounds became oval after wounding, and, thus, wound distances in the minor axis became short without a splint. In the present study and our previous study [10], the use of a splint decreased tension 


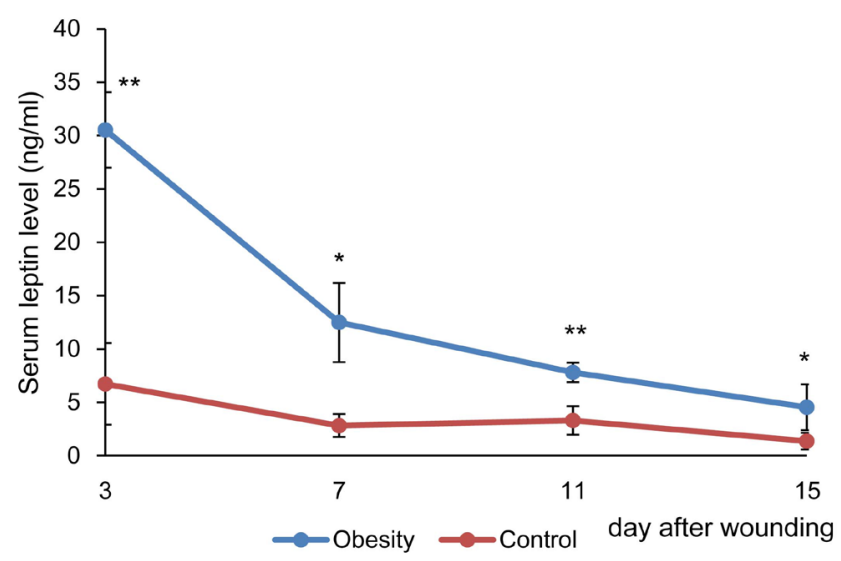

Figure 9. Serum leptin levels. Systemic leptin levels. Control group $n=3$, 5,3 , and 6 , Obesity group $n=3,3,3$, and 5 (on days $3,7,11$, and 15 after wounding, respectively). Values are means, with their standard deviation represented by vertical bars. The mean value was significantly different from that in the control group (unpaired $t$-test comparisons: ${ }^{\star} p<0.05$, $\left.{ }^{* *} p<0.01\right)$.

because it maintained the shape of the circular wound in obese mice. This new splinting method was effective for evaluating wound healing.

The amount of food consumed by obese mice markedly decreased after wounding. Consequently, mean body weights did not significantly differ between obese and non-obese mice. This may have been due to the stress induced by having $6-\mathrm{mm}$ wounds. Mean body weights were significantly higher in obese mice than in non-obese mice after wounding because the stress of wounding was decreased by changing to a circular wound size that was $4 \mathrm{~mm}$ in diameter. Therefore, we established experimental conditions to evaluate wound healing in obese mice without wound tension and the maintenance of body weight.

In spite of these experimental conditions, the progression of wound healing appeared to be similar in both groups for 15 days, with all wounds ultimately healing and forming scars on day 15 after wounding. This result indicates that the progression of cutaneous wound healing was not significantly different between obesity without hyperglycemia and non-obesity. Our results appear to differ from previous findings [34] [35].

In hypertrophied adipocytes, the expression of TNF- $\alpha$ is up-regulated. TNF- $\alpha$ is also produced by macrophages. Inflammatory responses were previously reported to be increased in a co-culture of adipocytes and macrophages [13], and the infiltration of macrophages was detected in adipose tissue from day 21 after the initiation of high-fat feeding and then in epididymal fatty tissue [38]. The number of macrophages in subcutaneous adipose tissue positively correlated with adipocyte size and body mass [39]. Furthermore, inflammation was enhanced in the adipose tissue of obese mice in these previous studies [21] [38] [39] [40]. On the other hand, our results showed no significant difference in the number of macrophages in subcutaneous adipose tissue between the control and obesity groups on the day before wounding. This result differed from previous 
findings showing that macrophages infiltrated adipose tissue 12 weeks after the initiation of a high-fat diet (56\% of calories from fat) [21]; 16 weeks and $60 \%$ [38], 12 weeks and $45 \%$ [39] and 15 weeks and 60\% [40]. We consider this to be one of the reasons why wound healing was not delayed in obese mice and indicates that if macrophages are not induced by numerous hypertrophied adipocytes under obese conditions, cutaneous wound healing in obese mice does not significantly differ from that in non-obese mice.

Topically applied leptin has been shown to accelerate wound healing by increasing keratinocyte proliferation [41] [42], enhancing angiogenesis [42] [43], and up-regulating collagen production by dermal fibroblasts [44]. In this study, we did not observe the blood leptin concentration on the day before wounding. However, at 15 weeks after feeding, the day before wounding, the leptin concentration in the obesity group $(42.3 \pm 3.5 \mathrm{ng} / \mathrm{mL})$ was significantly higher than that in the control group $(3.5 \pm 1.1 \mathrm{ng} / \mathrm{mL})$ [9]. In this study and our previous study, obese mice were fed the same diet for the same period. Therefore, in this study, serum leptin levels in the obesity group were also higher than that in the control group. Since serum levels of leptin in the present study were significantly higher in the obesity group than in the non-obese mouse until day 15 after wounding, its efficacy in cutaneous wound healing may have negated wound healing delays associated with obesity. Therefore, cutaneous wound healing was similar between obese and non-obese mice. A high serum level of leptin appears to promote cutaneous wound healing in obese mice.

Obesity is a major cause of type 2 DM [45], which clinically exhibits hyperglycemia [46] [47]. Previous studies reported wound healing delays in patients with DM [3] and a hyperglycemic state [25] [26]. Hyperglycemia affects re-epithelization [3] [26], neovascularization [3], and collagen accumulation [3] [25] [26] in wounds. Although our previous finding [9] and our present results showed that FBG levels were significantly higher in the obesity group than in the control group, FBG levels in our obese mouse model were lower than those in transgenic hyperglycemic models [27] [28] [48]. The ratio of myofibroblasts peaked on days 11 and 7 after wounding in the obesity and control groups, respectively. Wound contraction is mostly due to myofibroblasts [49]. Although the ratio of myofibroblasts in each group peaked on different days, the ratio of the wound area on days $7-15$ after wounding was not significantly different between the two groups. Therefore, a different peak of the ratio of myofibroblasts did not affect wound contraction in this study. Additionally, the ratio of collagen deposition and the numbers of macrophages, neutrophils, and new blood vessels in the obesity group did not also significantly differ from those in the control group. Therefore, undelayed wound healing was observed in the obesity group. This result indicates that hyperglycemia exerts stronger effects on wound healing than the excessive accumulation of adipose tissue. There is no evidence to demonstrate that obesity does not delay wound healing; therefore, further studies are warranted. 


\section{Conclusion}

Our hypothesis that obesity without hyperglycemia delays cutaneous wound healing is not correct because wound healing did not significantly differ between the obesity and control groups. Our results demonstrated that cutaneous wound healing was not delayed in the obesity group without hyperglycemia and macrophage infiltration into the subcutaneous fat and with high serum leptin levels. Our study also suggests that not all types of obesity delay wound healing; therefore, not all individuals with obesity will have delayed wound healing in clinical settings. We need to conduct further research to clarify why few macrophages infiltrated subcutaneous adipose tissue in our obese mouse model.

\section{Acknowledgements}

This work was supported by JSPS Grant-in-Aid for Scientific Research (B) Grant Number JP25293430. The funder had no role in study design, data collection and analysis, decision to publish, or preparation of the manuscript.

\section{Conflict of Interest}

None of the authors has any conflicts of interest to declare.

\section{References}

[1] Kanazawa, M., Yoshiike, N., Osaka, T., Numba, Y., Zimmet, P. and Inoue, S. (2002) Criteria and Classification of Obesity in Japan and Asia-Oceania. Asia Pacific Journal of Clinical Nutrition, 11, 732S-737S. https://doi.org/10.1046/j.1440-6047.11.s8.19.x

[2] Examination Committee of Criteria for "Obesity Disease" in Japan; Japan Society for the Study of Obesity. (2002) New Criteria for "Obesity Disease" in Japan. Circulation Journal, 66, 987-992.

[3] Guo, S. and Dipietro, L.A. (2010) Factors Affecting Wound Healing. Journal of Dental Research, 89, 219-229. https://doi.org/10.1177/0022034509359125

[4] Armstrong, M. (1998) Obesity as an Intrinsic Factor Affecting Wound Healing. Journal of Wound Care, 7, 220-221. https://doi.org/10.12968/jowc.1998.7.5.220

[5] Wilson, J.A. and Clark, J.J. (2004) Obesity: Impediment to Postsurgical Wound Healing. Advances in Skin \& Wound Care, 17, 426-435. https://doi.org/10.1097/00129334-200410000-00013

[6] Anaya, D.A. and Dellinger, E.P. (2006) The Obese Surgical Patient: A Susceptible Host for Infection. Surgical Infections (Larchmt), 7, 473-480.

https://doi.org/10.1089/sur.2006.7.473

[7] Sugerman, H., Windsor, A., Bessos, M. and Wolfe, L. (1997) Intra-Abdominal Pressure, Sagittal Abdominal Diameter and Obesity Comorbidity. Journal of Internal Medicine, 241, 71-79. https://doi.org/10.1046/j.1365-2796.1997.89104000.x

[8] Kabon, B., Nagele, A., Reddy, D., Eagon, C., Fleshman, J.W., Sessler, D.I. and Kurz, A. (2004) Obesity Decreases Perioperative Tissue Oxygenation. Anesthesiology, 100, 274-280. https://doi.org/10.1097/00000542-200402000-00015

[9] Urai, T., Haryanto, Mukai, K., Matsushita, T., Asano, K., Nakajima, Y., Okuwa, M., Sugama, J. and Nakatani, T. (2016) The Relationship between Cutaneous Wounds 
Made on Obese Mice or Those with Decreased Body Weight and Serum Leptin Level. Health, 8, 1015-1028. https://doi.org/10.4236/health.2016.811105

[10] Urai, T., Asano, K., Nakajima, Y., Mukai, K. and Nakatani, T. (2017) Effectiveness of Using an Ostomy Skin Barrier/wafer as a Splint to Maintain the Shapes of Circular Cutaneous Wounds in Obese Mice. Structure and Function, 15, 57-61. (In Japanese with English Abstract)

[11] Abranches, M.V., de Oliveira, F.C.E., da Conceição, L.L. and Peluzio, M.do.C.G. (2015) Obesity and Diabetes: The Link between Adipose Tissue Dysfunction and Glucose Homeostasis. Nutrition Research Reviews, 28, 121-132. https://doi.org/10.1017/S0954422415000098

[12] Suganami, T. and Ogawa, Y. (2010) Adipose Tissue Macrophages: Their Role in Adipose Tissue Remodeling. Journal of Leukocyte Biology, 88, 33-39. https://doi.org/10.1189/jlb.0210072

[13] Suganami, T., Nishida, J. and Ogawa, Y. (2005) A Paracrine Loop between Adipocytes and Macrophages Aggravates Inflammatory Changes: Role of Free Fatty Acids and Tumor Necrosis Factor a. Arteriosclerosis, Thrombosis, and Vascular Biology, 25, 2062-2068. https://doi.org/10.1161/01.ATV.0000183883.72263.13

[14] Schenk, S., Saberi, M. and Olefsky, J.M. (2008) Insulin Sensitivity: Modulation by Nutrients and Inflammation. The Journal of Clinical Investigation, 118, 2992-3002. https://doi.org/10.1172/JCI34260

[15] Wajchenberg, B.L. (2000) Subcutaneous and Visceral Adipose Tissue: Their Relation to the Metabolic Syndrome. Endocrine Reviews, 21, 697-738. https://doi.org/10.1210/edrv.21.6.0415

[16] Cottam, D.R., Mattar, S.G., Barinas-Mitchell, E., Eid, G., Kuller, L., Kelley, D.E. and Schauer, P.R. (2004) The Chronic Inflammatory Hypothesis for the Morbidity Associated with Morbid Obesity: Implications and Effects of Weight Loss. Obesity Surgery, 14, 589-600. https://doi.org/10.1381/096089204323093345

[17] Sun, K., Kusminski, C.M. and Scherer, P.E. (2011) Adipose Tissue Remodeling and Obesity. The Journal of Clinical Investigation, 121, 2094-2101. https://doi.org/10.1172/JCI45887

[18] Rivera-Gonzalez, G., Shook, B. and Horsley, V. (2014) Adipocytes in Skin Health and Disease. Cold Spring Harbor Perspectives in Medicine, 4, a015271.

[19] Yamauchi, T., Kamon, J., Waki, H., Terauchi, Y., Kubota, N., Hara, K., Mori, Y., Ide, T., Murakami, K., Tsuboyama-Kasaoka, N., Ezaki, O., Akanuma, Y., Gavrilova, O., Vinson, C., Reitman, M.L., Kagechika, H., Shudo, K., Yoda, M., Nakano, Y., Tobe, K., Nagai, R., Kimura, S., Tomita, M., Froguel, P. and Kadowaki, T. (2001) The Fat-Derived Hormone Adiponectin Reverses Insulin Resistance Associated with Both Lipoatrophy and Obesity. Nature Medicine, 7, 941-946.

https://doi.org/10.1038/90984

[20] Awazawa, M., Ueki, K., Inabe, K., Yamauchi, T., Kubota, N., Kaneko, K., Kobayashi, M., Iwane, A., Sasako, T., Okazaki, Y., Ohsugi, M., Takamoto, I., Yamashita, S., Asahara, H., Akira, S., Kasuga, M. and Kadowaki, T. (2011) Adiponectin Enhances Insulin Sensitivity by Increasing Hepatic IRS-2 Expression via a Macrophage-Derived IL-6-Dependent Pathway. Cell Metabolism, 13, 401-412. https://doi.org/10.1016/j.cmet.2011.02.010

[21] Kanda, H., Tateya, S., Tamori, Y., Kotani, K., Hiasa, K., Kitazawa, R., Kitazawa, S., Miyachi, H., Maeda, S., Egashira, K. and Kasuga, M. (2006) MCP-1 Contributes to Macrophage Infiltration into Adipose Tissue, Insulin Resistance, and Hepatic Steatosis in Obesity. The Journal of Clinical Investigation, 116, 1494-1505. 
https://doi.org/10.1172/JCI26498

[22] Liu, C., Feng, X., Li, Q., Wang, Y., Li, Q. and Hua, M. (2016) Adiponectin, TNF- $\alpha$ and Inflammatory Cytokines and Risk of Type 2 Diabetes: A Systematic Review and Meta-Analysis. Cytokine, 86, 100-109. https://doi.org/10.1016/j.cyto.2016.06.028

[23] Yoshiike, N., Nishi, N., Matsushima, S., Itou, C., Ikeda, Y., Kashihara, H., Yoshinaga, H., Ogura, H., Komine, S., Satou, Y., Satoh, N., Sasaki, A., Fujioka, S., Oku, J., Amemiya, S., Sakata, T. and Inoue, S. (2000) Relation between Body Mass Index and Risk for Diabetes, Hypertension, Hyperlipidemia-An Epidemiological Study by Multicenter Cooperative Study. HimanKenkyu, 6, 4-17. (In Japanese)

[24] Jerant, A., Bertakis, K.D. and Franks, P. (2015) Body Mass Index and Health Care Utilization in Diabetic and Nondiabetic Individuals. Medical Care, 53, 409-416. https://doi.org/10.1097/MLR.0000000000000343

[25] Goodson, W.H. and Hunt, T.K. (1986) Wound Collagen Accumulation in Obese Hyperglycemic Mice. Diabetes, 35, 491-495. https://doi.org/10.2337/diab.35.4.491

[26] Huang, L., Nakagami, G., Minematsu, T., Kinoshita, A., Sugama, J., Nakatani, T., Sagara, H. and Sanada, H. (2010) Ulceration and Delayed Healing Following Pressure Loading in Hyperglycemic Rats with an Immature Dermal Collagen Fiber Network. Wounds, 22, 237-244.

[27] Ingalls, A.M., Dickie, M.M. and Snell, G. (1950) Obese, a New Mutation in the House Mouse. Journal of Heredity, 41, 317-318. https://doi.org/10.1093/oxfordjournals.jhered.a106073

[28] Hummel, K.P., Dickie, M.M. and Coleman, D.L. (1966) Diabetes, a New Mutation in the Mouse. Science, 153, 1127-1128. https://doi.org/10.1126/science.153.3740.1127

[29] Jetten, N., Roumans, N., Gijbels, M.J., Romano, A., Post, M.J., De Winther, M.P.J., Van Der Hulst, R.R.W.J. and Xanthoulea, S. (2014) Wound Administration of M2-Polarized Macrophages Does Not Improve Murine Cutaneous Healing Responses. PLoS ONE, 9, e102994. https://doi.org/10.1371/journal.pone.0102994

[30] Brown, D.L., Kao, W.W.Y. and Greenhalgh, D.G. (1997) Apoptosis Down-Regulates Inflammation under the Advancing Epithelial Wound Edge: Delayed Patterns in Diabetes and Improvement with Topical Growth Factors. Surgery, 121, 372-380. https://doi.org/10.1016/S0039-6060(97)90306-8

[31] Seitz, O., Schurmann, C., Hermes, N., Muller, E., Pfeilschifter, J., Frank, S. and Goren, I. (2010) Wound Healing in Mice with High-Fat Diet- or $o b$ Gene-Induced Diabetes-Obesity Syndromes: A Comparative Study. Experimental Diabetes Research, 2010, Article ID: 476969.

[32] Cefalu, W.T. (2006) Animal Models of Type 2 Diabetes: Clinical Presentation and Pathophysiological Relevance to the Human Condition. ILAR Journal, 47, 186-198. https://doi.org/10.1093/ilar.47.3.186

[33] Rees, D.A. and Alcolado, J.C. (2005) Animal Models of Diabetes Mellitus. Diabetic Medicine, 22, 359-370. https://doi.org/10.1111/j.1464-5491.2005.01499.x

[34] Nascimento, A.P. and Costa, A.M. (2006) Overweight Induced by High-Fat Diet Delays Rat Cutaneous Wound Healing. British Journal of Nutrition, 96, 1069-1077. https://doi.org/10.1017/BJN20061955

[35] Nascimento, A.P. and Costa, A.M. (2011) Both Obesity-Prone and Obesity-Resistant Rats Present Delayed Cutaneous Wound Healing. British Journal of Nutrition, 106, 603-611. https://doi.org/10.1017/S0007114511000468

[36] Nakajima, Y., Nakano, Y., Fuwano, S., Hayashi, N., Hiratoko, Y., Kinoshita, A., 
Miyahara, M., Mochizuki, T., Nishino, K., Tsuruhara, Y., Yokokawa, Y., Iuchi, T., Kon, Y., Mukai, K., Kitayama, Y., Murakado, N., Okuwa, M. and Nakatani, T. (2013) Effects of Three Types of Japanese Honey on Full-Thickness Wound in Mice. Evidence-Based Complementary and Alternative Medicine, 2013, Article ID: 504537. https://doi.org/10.1155/2013/504537

[37] Mukai, K., Koike, M., Nakamura, S., Kawaguchi, Y., Katagiri, F., Nojiri, S., Yamada, Y., Miyajima, E., Matsumoto, M., Komatsu, E., Nakajima, Y., Urai, T., Murakado, N. and Nakatani, T. (2015) Evaluation of the Effects of a Combination of Japanese Honey and Hydrocolloid Dressing on Cutaneous Wound Healing in Male Mice. Evidence-Based Complementary and Alternative Medicine, 2015, Article ID: 910605. https://doi.org/10.1155/2015/910605

[38] Elgazar-Carmon, V., Rudich, A., Hadad, N. and Levy, R. (2008) Neutrophils Transiently Infiltrate Intra-Abdominal Fat Early in the Course of High-Fat Feeding. The Journal of Lipid Research, 49, 1894-1903. https://doi.org/10.1194/jlr.M800132-JLR200

[39] Weisberg, S.P., McCann, D., Desai, M., Rosenbaum, M., Leibel, R.L. and Ferrante, A.W. (2003) Obesity Is Associated with Macrophage Accumulation in Adipose Tissue. The Journal of Clinical Investigation, 112, 1796-1808. https://doi.org/10.1172/JCI200319246

[40] Ito, A., Suganami, T., Miyamoto, Y., Yoshimasa, Y., Takeya, M., Kamei, Y. and Ogawa, Y. (2007) Role of MAPK Phosphatase-1 in the Induction of Monocyte Chemoattractant Protein-1 during the Course of Adipocyte Hypertrophy. The Journal of Biological Chemistry, 282, 25445-25452.

https://doi.org/10.1074/jbc.M701549200

[41] Frank, S., Stallmeyer, B., Kämpfer, H., Kolb, N. and Pfeilschifter, J. (2000) Leptin Enhances Wound Re-Epithelialization and Constitutes a Direct Function of Leptin in Skin Repair. The Journal of Clinical Investigation, 106, 501-509. https://doi.org/10.1172/JCI9148

[42] Tadokoro, S., Ide, S., Tokuyama, R., Umeki, H., Tatehara, S., Kataoka, S., Satomura, K. (2015) Leptin Promotes Wound Healing in the Skin. PLoS ONE, 10, e0121242. https://doi.org/10.1371/journal.pone.0121242

[43] Murad, A., Nath, A.K., Cha, S.T., Demir, E., Flores-Riveros, J. and Sierra-Honigmann, M.R. (2003) Leptin Is an Autocrine/Paracrine Regulator of Wound Healing. The FASEB Journal, 17, 1895-1897. https://doi.org/10.1096/fj.03-0068fje

[44] Ezure, T. and Amano, S. (2007) Adiponectin and Leptin Up-Regulate Extracellular Matrix Production by Dermal Fibroblasts. Biofactors, 31, 229-236. https://doi.org/10.1002/biof.5520310310

[45] Martyn, J.A.J., Kaneki, M. and Yasuhara, S. (2008) Obesity-Induced Insulin Resistance and Hyperglycemia: Etiologic Factors and Molecular Mechanisms. Anesthesiology, 109, 137-148. https://doi.org/10.1097/ALN.0b013e3181799d45

[46] Chan, J.M., Rimm, E.B., Colditz, G.A., Stampfer, M.J. and Willett, W.C. (1994) Obesity, Fat Distribution, and Weight Gain as Risk Factors for Clinical Diabetes in Men. Diabetes Care, 17, 961-969. https://doi.org/10.2337/diacare.17.9.961

[47] Colditz, G.A., Willett, W.C., Rotnitzky, A. and Manson, J.E. (1995) Weight Gain as a Risk Factor for Clinical Diabetes Mellitus in Women. Annals of Internal Medicine, 122, 481-486. https://doi.org/10.7326/0003-4819-122-7-199504010-00001

[48] Goto, Y., Kakizaki, M. and Masaki, N. (1976) Production of Spontaneous Diabetic Rats by Repetition of Selective Breeding. The Tohoku Journal of Experimental Medicine, 119, 85-90. https://doi.org/10.1620/tjem.119.85 
[49] Broughton II, G., Janis, J.E. and Attinger, C.E. (2006) The Basic Science of Wound Healing. Plastic and Reconstructive Surgery, 117, 12S-34S.

https://doi.org/10.1097/01.prs.0000225430.42531.c2 